\title{
GGHLite: More Efficient Multilinear Maps from Ideal Lattices
}

\author{
Adeline Langlois ${ }^{1}$, Damien Stehlé ${ }^{1}$, and Ron Steinfeld ${ }^{2}$ \\ 1 ENS de Lyon, Laboratoire LIP (U. Lyon, CNRS, ENS Lyon, INRIA, UCBL), \\ 46 Allée d'Italie, 69364 Lyon Cedex 07, France \\ 2 Clayton School of Information Technology, Monash University, Clayton, Australia
}

\begin{abstract}
The GGH Graded Encoding Scheme [9, based on ideal lattices, is the first plausible approximation to a cryptographic multilinear map. Unfortunately, using the security analysis in 9], the scheme requires very large parameters to provide security for its underlying "encoding re-randomization" process. Our main contributions are to formalize, simplify and improve the efficiency and the security analysis of the re-randomization process in the GGH construction. This results in a new construction that we call GGHLite. In particular, we first lower the size of a standard deviation parameter of the re-randomization process of 9 from exponential to polynomial in the security parameter. This first improvement is obtained via a finer security analysis of the "drowning" step of re-randomization, in which we apply the Rényi divergence instead of the conventional statistical distance as a measure of distance between distributions. Our second improvement is to reduce the number of randomizers needed from $\Omega(n \log n)$ to 2 , where $n$ is the dimension of the underlying ideal lattices. These two contributions allow us to decrease the bit size of the public parameters from $O\left(\lambda^{5} \log \lambda\right)$ for the GGH scheme to $O\left(\lambda \log ^{2} \lambda\right.$ ) in GGHLite, with respect to the security parameter $\lambda$ (for a constant multilinearity parameter $\kappa$ ).
\end{abstract}

\section{Introduction}

Boneh and Silverberg [6] defined a cryptographic $\kappa$-multilinear map e as a map from $G_{1} \times \ldots \times G_{\kappa}$ to $G_{T}$, all cyclic groups of order $p$, which enjoys three main properties: first, for any elements $g_{i} \in G_{i}$ for $i \leq \kappa, j \leq \kappa$ and $\alpha \in$ $\mathbb{Z}_{p}$, we have $e\left(g_{1}, \ldots, \alpha \cdot g_{j}, \ldots, g_{\kappa}\right)=\alpha \cdot e\left(g_{1}, \ldots, g_{\kappa}\right)$; second, the map $e$ is non-degenerate, i.e., if the $g_{i}$ 's are generators of their respective $G_{i}$ 's then $e\left(g_{1}, \ldots, g_{\kappa}\right)$ generates $G_{T}$; and third, there is no efficient algorithm to compute discrete logarithms in any of the $G_{i}$ 's. Bilinear maps $(\kappa=2)$ and multilinear maps have a lot of cryptographic applications, see [11215] and 6201619], respectively. But unlike bilinear maps, built with pairings on elliptic curves, the construction of cryptographic multilinear maps was an open problem for several years. In [6], Boneh and Silverberg studied the interest of such maps, and gave two applications: multipartite Diffie-Hellman key exchange and very efficient broadcast encryption. But they conjectured that multilinear maps will probably "come from outside the realm of algebraic geometry." In 2013, Garg, Gentry

P.Q. Nguyen and E. Oswald (Eds.): EUROCRYPT 2014, LNCS 8441, pp. 239-256, 2014.

(C) International Association for Cryptologic Research 2014 
and Halevi 9 introduced the first "approximate" multilinear maps contruction, based on ideal lattices, and the powerful notion of graded encoding scheme. Based on their work, Coron, Lepoint and Tibouchi [7] recently described an alternative construction of graded encoding scheme.

We first give a high level description of the GGH graded encoding scheme 9]. If we come back to the definition of cryptographic multilinear maps, the authors of [9] notice that $\alpha \cdot g_{i}$ can be viewed as an "encoding" of the "plaintext" $\alpha \in \mathbb{Z}_{q}$. They consider the polynomial rings $R=\mathbb{Z}[x] /\left\langle x^{n}+1\right\rangle$ and $R_{q}=R / q R$ (replacing the exponent space $\mathbb{Z}_{p}$ ). They generate a small secret $g \in R$ and let $\mathcal{I}=\langle g\rangle$ be the principal ideal over $R$ generated by $g$. They also sample a uniform $z \in R_{q}$ which stays secret. The "plaintext" is an element of $R / \mathcal{I}$, and is encoded via a division by $z$ in $R_{q}$ : to encode a coset of $R / \mathcal{I}$, return $[c / z]_{q}$, where $c$ is an arbitrary small coset representative. In practice, as $g$ is hidden, they give another public parameter $y$, which is an encoding of 1 , and the encoding of the coset is computed as $[e \cdot y]_{q}$, where $e$ is a small coset representative (possibly different from $c$ ). But, as opposed to multilinear maps, their graded encoding scheme uses the notion of encoding level: the plaintext $e$ is a level-0 encoding, the encoding $[c / z]_{q}$ is a level-1 encoding, and at level $i$, an encoding of $e+\mathcal{I}$ is given by $\left[c / z^{i}\right]_{q}=\left[e \cdot y^{i}\right]_{q}$. These encodings are both additively and multiplicatively homomorphic, up to a limited number of operations. More precisely, a product of $i$ level-1 encodings is a level- $i$ encoding. One can multiply any number of encodings up to $\kappa$, instead of exactly $\kappa$ in multilinear maps (the parameter $\kappa$ is called the multilinearity parameter).

The authors of [9] introduced new hardness assumptions: the Graded Decisional Diffie-Hellman (GDDH) and its computational variant (GCDH). These are natural analogues of the Diffie-Hellman problems from group-based cryptography. To ensure their hardness, and hence the security of the cryptographic constructions, the second main difference with multilinear maps is the randomization of the encodings. The principle is as follows: first some level-1 encodings of 0 , called $\left\{x_{j}=\left[b_{j} / z\right]_{q}\right\}_{j \leq m_{r}}$, are given as part of the public parameters; then, to randomize a level-1 encoding $u^{\prime}=[e \cdot y]_{q}$, one outputs $u=\left[u^{\prime}+\sum_{j} \rho_{j} x_{j}\right]_{q}=[c / z]_{q}$ with $c=c^{\prime}+\sum_{j} \rho_{j} b_{j}$, where the $\rho_{j}$ 's are sampled from a discrete Gaussian distribution over $\mathbb{Z}$ with deviation parameter $\sigma^{*}$. Without this re-randomization, the encoding $u^{\prime}$ of $e$ allows $e$ to be efficiently recovered using $u=\left[u^{\prime} y^{-1}\right]_{q}$. Adding the re-randomization step prevents this division attack, but the statistical properties of the distribution of the re-randomized encoding $u$ remain correlated to some extent with the original encoding $u^{\prime}$ (for instance, the center of the distribution of $c$ is $c^{\prime}$, since the distribution of $\sum_{j} \rho_{j} b_{j}$ is known to be centered at 0 ). This property may allow other attacks that exploit this correlation. The question arises as to how to set the re-randomization parameter $\sigma^{*}$ in order to guarantee security against such potential "statistical correlation" attacks - the larger the re-randomization parameters the smaller the correlation, and heuristically the more resistant the scheme is to such attacks. But increasing $\sigma^{*}$ impacts the efficiency of the scheme. 
In [9], the authors use a "drowning step" to solve this problem. This technique, also called "smudging," was previously used in other applications [31024]. Generally, "drowning" consists in hiding a secret vector $s \in \mathbb{Z}^{n}$ by adding a sufficiently large random noise $\boldsymbol{e} \in \mathbb{Z}^{n}$ to it, so that the distribution of $\boldsymbol{s}+\boldsymbol{e}$ becomes "almost independent" of $\boldsymbol{s}$. In all of the above applications, to achieve a security level $2^{\lambda}$ (where $\lambda$ denotes the security parameter), the security analysis requires "almost independent" to be interpreted as "within statistical distance $2^{-\lambda}$ from a distribution that is independent of $s$." In turn, this requirement implies the need for "exponential drowning," i.e., the ratio $\gamma=\|\boldsymbol{e}\| /\|\boldsymbol{s}\|$ between the magnitude of the noise and the magnitude of secret needs to be $2^{\Omega(\lambda)}$. Exponential drowning imposes a severe penalty on the efficiency of these schemes, as their security is related to $\gamma$-approximation lattice problems, whose complexity decreases exponentially with $\log \gamma$. As a result, the schemes require a lattice dimension $n$ at least quadratic in $\lambda$ and key length at least cubic in $\lambda$. In summary, the GGH re-randomization step, necessary for its security, is also a primary factor in its inefficiency.

OUR CONTRIBUTIONS. First, we formalize the re-randomization security goal in the GGH construction, that is implicit in the work of [9]. A primary security goal of re-randomization is to guarantee security of the GDDH problem against statistical correlation attacks. Accordingly, we formulate a security goal that captures this security guarantee, by introducing a canonical variant of GDDH, called cGDDH. In this variant, the encodings of some elements are sampled from a canonical distribution whose statistical properties are independent of the encoded elements. Consequently, the canonical problems are by construction not subject to "statistical correlation" attacks. Our re-randomization security goal is formulated as the existence of an efficient computational reduction from the canonical problems to their corresponding non-canonical variants.

Our first main improvement to the GGH scheme relies on a new security analysis of the drowning step in the GGH re-randomization algorithm. We show that our re-randomization security goal can be satisfied without "exponential drowning," thus removing the main efficiency bottleneck. Namely, our analysis provides a re-randomization at security level $2^{\lambda}$ while allowing the use of a re-randomization deviation parameter $\sigma^{*}$ that only drowns the norm of the randomness offset $r^{\prime} \in \mathcal{I}$ (from the original encoding to be re-randomized) by a polynomial (or even constant) drowning ratio $\gamma=\lambda^{O(1)}$ (rather than $\gamma=2^{\Omega(\lambda)}$, as needed in the analysis of [9]). However, our analysis only works for the search variant of the Graded Diffie-Hellman problem. Fortunately, we show that the two flagship applications of the GGH scheme - the $N$-party Key Agreement and the Attribute Based Encryption - can be modified to rely on this computational assumption (in the random oracle model).

Our second main improvement of the re-randomization process is to decrease $m_{r}$, the number of encodings of 0 needed, from $\Omega(n \log n)$ to 2 . We achieve this result by presenting a new discrete Gaussian Leftover Hash Lemma (LHL) over algebraic rings. In [9], the authors apply the discrete Gaussian LHL from 1] to show that the distribution of the sum $\sum_{j \leq m_{r}} \rho_{j} r_{j}$ is close to a discrete Gaussian 
on the ideal $\mathcal{I}$. Our improvement consists in sampling the randomizers $\rho_{j}$ as elements of the full $n$-dimensional ring $R$, rather than just from $\mathbb{Z}$. Since each randomizer now has $n$ times more entropy than before, one may hope to obtain a similar LHL result as in [1] while reducing $m_{r}$ by a factor $\approx n$. However, as the designers of the GGH scheme notice in [9, Se. 6.4], the proof techniques from [1] do not seem to immediately carry over to our "algebraic ring" LHL setting. Our new LHL over rings resolves this problem.

These contributions allow us to decrease the bit size of the public parameters from $O\left(\kappa^{3} \lambda^{5} \log (\kappa \lambda)\right)$ for the GGH scheme to $O\left(\kappa^{2} \lambda \log ^{2}(\kappa \lambda)\right)$ for GGHLite, for security level $2^{\lambda}$ for the graded Diffie-Hellman problem.

TECHNiCAL OVERVIEW. Our first main result is to reduce the size of the parameter $\sigma^{*}$ in the re-randomization process. Technically, our improved analysis of drowning is obtained by using the Rényi divergence (RD) to replace the conventional statistical distance (SD) as a measure of distribution closeness. The RD was already exploited in a different context in [13, Claim 5.11], to show the hardness of Ring-LWE. Here, we use the RD to decrease the amount of drowning, by bounding the RD between a discrete Gaussian distribution and its offset.This suffices for relating the hardness of the search problems using these encoding distributions, even though the SD between the distributions is non-negligible. The technique does not seem to easily extend to the decision problems, as RD induces a multiplicative relationship between success probabilities, rather than an additive relationship as SD does.

Our second main result is a new LHL over the ring $R$. We now briefly explain this result and its proof. For a fixed $X=\left[x_{1}, x_{2}\right] \in R^{2}$, with each $x_{i}$ sampled from $D_{R, s}$, our goal is to study the distribution $\widetilde{\mathcal{E}}_{X, s}=x_{1} \cdot D_{R, s}+x_{2} \cdot D_{R, s}$. In particular, we prove that $\widetilde{\mathcal{E}}_{X, s}$ is statistically close to $D_{\mathbb{Z}^{n}, s X^{T}}$. For this, we adapt the proof of the LHL in [1]: we follow a similar series of steps, but the proofs of these steps differ technically, as we exploit the ring structure.

We first show that $X \cdot R^{2}=R$, except with some constant probability $<1$. For this, we adapt a result from 23 . on the probability that two Gaussian samples of $R$ are coprime. Note that in contrast to the LHL over $\mathbb{Z}$ in [1], in our setting the probability that $X \cdot R^{2} \neq R$ is non-negligible. This is unavoidable with the ring $R=\mathbb{Z}[x] /\left\langle x^{n}+1\right\rangle$, since each random element of $R$ falls in the ideal $\langle x+1\rangle$ with probability $\approx 1 / 2$, both $x_{1}$ and $x_{2}$ (and hence the ideal they generate) get "stuck" in $\langle x+1\rangle$ with probability $\approx 1 / 4$. However, the probability of this bad event is bounded away from 1 by a constant and thus we only need a constant number of trials on average with random $X$ 's to obtain a good $X$ by rejection.

Then, we define the orthogonal $R$-module $A_{X}=\left\{\boldsymbol{v} \in R^{2}: X \cdot \boldsymbol{v}=0\right\}$, and apply a directly adapted variant of [1, Le. 10] to show that if the parameter $s$ is larger than the smoothing parameter $\eta_{\varepsilon}\left(A_{X}\right)$ (with $A_{X}$ viewed as an integral lattice), then the SD between $\widetilde{\mathcal{E}}_{X, s}$ and the ellipsoidal Gaussian $D_{\mathbb{Z}^{n}, s X^{T}}$ is bounded by $2 \varepsilon$. We finally show that this condition on the smoothing parameter of $A_{X}$ holds. For this, we observe that the Minkowski minima of the lattice $A_{X}$ are equal, due to the $R$-module structure of $A_{X}$. This allows us to bound the last minimum from above using Minkowski's second theorem. A similar approach 
was previously used (e.g., in [12]) to bound the smoothing parameter of ideal lattices.

Notation. A function $f(\lambda)$ is said negligible if it is $\lambda^{-\omega(1)}$. For an integer $q$, we let $\mathbb{Z}_{q}$ denote the ring of integers modulo $q$. The notation $[\cdot]_{q}$ means that all operations within the square brackets are performed modulo $q$. We choose $n \geq 4$ as a power of 2 , and let $K$ and $R$ respectively denote the polynomial ring $\mathbb{Q}[X] /\left\langle x^{n}+\right.$ $1\rangle$ and $\mathbb{Z}[X] /\left\langle x^{n}+1\right\rangle$. The rings $K$ and $R$ are isomorphic to the cyclotomic field of order $2 n$ and its ring of integers, respectively. For an integer $q$, we let $R_{q}$ denote the ring $\mathbb{Z}_{q}[x] /\left\langle x^{n}+1\right\rangle \simeq R / q R$. For $z \in R$ we denote by $\operatorname{MSB}_{\ell}(z) \in\{0,1\}^{\ell \cdot n}$ the $\ell$ most-significant bits of each of the $n$ coefficients of $z$. Vectors are denoted in bold. For $\boldsymbol{b} \in \mathbb{R}^{d}$ (resp. $g \in K$ ), we let $\|\boldsymbol{b}\|$ (resp. $\|g\|$ ) denote its Euclidean norm (resp. norm of its coefficient vector). The uniform distribution on finite set $E$ is denoted by $U(E)$. The statistical distance (SD) between distributions $D_{1}$ and $D_{2}$ over a countable domain $E$ is $\frac{1}{2} \sum_{x \in E}\left|D_{1}(x)-D_{2}(x)\right|$. For a function $f$ over a countable domain $E$, we let $f(E)=\sum_{x \in E} f(x)$. Let $X \in \mathbb{R}^{m \times n}$ be a rank- $n$ matrix and $U_{X}=\left\{\|X \boldsymbol{u}\|: \boldsymbol{u} \in \mathbb{R}^{n},\|\boldsymbol{u}\|=1\right\}$. The smallest (resp. largest) singular value of $X$ is denoted by $\sigma_{n}(X)=\inf \left(U_{X}\right)\left(\operatorname{resp} . \sigma_{1}(X)=\sup \left(U_{X}\right)\right)$.

REMARK. Due to lack of space, some contents have been postponed to the full version of this paper, available from the webpages of the authors.

\section{Preliminaries}

Lattices. We refer to [1417] for introductions to the computational aspects of lattices. A $d$-dimensional lattice $\Lambda \subseteq \mathbb{R}^{n}$ is the set of all integer linear combinations $\sum_{i=1}^{d} x_{i} \boldsymbol{b}_{i}$ of some linearly independent vectors $\boldsymbol{b}_{i} \in \mathbb{R}^{n}$. The determinant $\operatorname{det}(\Lambda)$ is defined as $\sqrt{\operatorname{det}\left(B^{T} B\right)}$, where $B=\left(\boldsymbol{b}_{i}\right)_{i}$ is any such basis of $\Lambda$. For $i \leq d$, the $i$ th minimum $\lambda_{i}(\Lambda)$ is the smallest $r$ such that $\Lambda$ contains $i$ linearly independent vectors of norms $\leq r$.

Gaussian Distributions. For a rank- $n$ matrix $S \in \mathbb{R}^{m \times n}$ and a vector $c \in \mathbb{R}^{n}$, the ellipsoid Gaussian distribution with parameter $S$ and center $c$ is defined as: $\forall \boldsymbol{x} \in \mathbb{R}^{n}, \rho_{S, \boldsymbol{c}}(x)=\exp \left(-\pi(\boldsymbol{x}-\boldsymbol{c})^{T}\left(S^{T} S\right)^{-1}(\boldsymbol{x}-\boldsymbol{c})\right)$. Note that $\rho_{S, \boldsymbol{c}}(x)=$ $\exp \left(-\pi\left\|\left(S^{T}\right)^{\dagger}(\boldsymbol{x}-\boldsymbol{c})\right\|\right)$, where $X^{\dagger}$ denotes the pseudo-inverse of $X$. The ellipsoid discrete Gaussian distribution over a coset $\Lambda+z$ of a lattice $\Lambda$, with parameter $S$ and center $\boldsymbol{c}$ is defined as: $\forall \boldsymbol{x} \in \Lambda+z, D_{\Lambda+z, S, \boldsymbol{c}}=\rho_{S, \boldsymbol{c}}(\boldsymbol{x}) / \rho_{S, \boldsymbol{c}}(\Lambda)$.

Smoothing Parameter. Introduced by [15], the smoothing parameter $\eta_{\varepsilon}(\Lambda)$ of an $n$-dimensional lattice $\Lambda$ and a real $\varepsilon>0$ is defined as the smallest $s$ such that $\rho_{1 / s}\left(\Lambda^{*} \backslash\{0\}\right) \leq \varepsilon$. We use the following properties.

Lemma 2.1 ([15, Le. 3.3]). Let $\Lambda$ be an $n$-dimensional lattice and $\varepsilon>0$. Then $\eta_{\varepsilon}(\Lambda) \leq \sqrt{\ln (2 n(1+1 / \varepsilon)) / \pi} \cdot \lambda_{n}(\Lambda)$.

Lemma 2.2 ([1, Le. 3]). For a rank-n lattice $\Lambda$, constant $0<\varepsilon<1$, vector $\boldsymbol{c}$ and matrix $S$ with $\sigma_{n}(S) \geq \eta_{\varepsilon}(\Lambda)$, if $\boldsymbol{x}$ is sampled from $D_{\Lambda, S, \boldsymbol{c}}$ then $\|\boldsymbol{x}\| \leq$ $\sigma_{1}(S) \sqrt{n}$, except with probability $\leq \frac{1+\varepsilon}{1-\varepsilon} \cdot 2^{-n}$. 
Algebraic Number Rings and Ideal Lattices. For $g, x \in R$, we let $[x]_{g}$ denote the reduction of $x$ modulo the principal ideal $I=\langle g\rangle$ with respect to the $\mathbb{Z}$-basis $\left(g, x \cdot g, \ldots, x^{n-1} \cdot g\right)$, i.e., $[x]_{g}$ is the unique element of $R$ in $\mathcal{P}_{g}=$ $\left\{\sum_{i=0}^{n-1} c_{i} x^{i} g: c_{i} \in[-1 / 2,1 / 2) \cap \mathbb{R}\right\}$ such that $x-[x]_{g} \in\langle g\rangle$. The set $\mathcal{P}_{g} \cap R$ is a set of unique representatives of the cosets of $I$ in $R$, that make up the quotient ring $R / I$. To use our improved drowning lemma in Section 4 , we need a lower bound on the last singular value $\sigma_{n}(\operatorname{rot}(b))$ of the matrix $\operatorname{rot}(b) \in \mathbb{Z}^{n \times n}$ corresponding to the map $x \mapsto b \cdot x$ over $R$, for a Gaussian distributed $b \hookleftarrow D_{I, \sigma}$. In the following, and in the rest of the paper, we abuse notation and write $b$ for this matrix.

Lemma 2.3 (Adapted from [23, Le. 4.1]). Let $R=\mathbb{Z}^{n}[x] /\left(x^{n}+1\right)$ for $n a$ power of 2. For any ideal $I \subseteq R, \delta \in(0,1), t \geq \sqrt{2 \pi}$ and $\sigma \geq \frac{t}{\sqrt{2 \pi}} \cdot \eta_{\delta}(I)$, we have:

$$
\operatorname{Pr}_{b \hookleftarrow D_{I, \sigma}}\left[\left\|b^{-1}\right\| \geq \frac{t}{\sigma \sqrt{n / 2}}\right] \leq \operatorname{Pr}_{b \hookleftarrow D_{I, \sigma}}\left[\sigma_{n}(b) \leq \frac{\sigma \sqrt{n / 2}}{t}\right] \leq \frac{1+\delta}{1-\delta} \frac{n \sqrt{2 \pi \mathrm{e}}}{t} .
$$

\section{GGH and Its Re-randomization Procedure}

In this section, we recall the Garg et al. scheme from [9], and its related hard problems. We then discuss the re-randomization step of the scheme and explain what should be expected from it, in terms of security. This security requirement is unclear in [9] and [1]. We formulate it precisely. This will drive our re-randomization design in the following sections.

\subsection{The GGH Scheme}

We recall the GGH scheme in Figure 1. We present it here in a slightly more general form than [9]: we leave as a parameter the distribution $\chi_{k}$ of the rerandomization coefficients $\rho_{j}$ for a level- $k$ encoding (for any $k \leq \kappa$ ). In the original GGH scheme, we have $\chi_{k}=D_{\mathbb{Z}, \sigma_{k}^{*}}$ for some $\sigma_{k}^{*}$ 's, i.e., the $\rho_{j}$ 's are integers sampled from a discrete Gaussian distribution. Looking ahead, in Section [5] we analyze a more efficient variant, in which $\chi_{k}=D_{R, \sigma_{k}^{*}}$, so that the $\rho_{j}$ 's belong to $R$.

The aim of isZero is to test whether the input $u=\left[c / z^{\kappa}\right]_{q}$ is a level- $\kappa$ encoding of 0 or not, i.e., whether $c=g \cdot r$ for some $r \in R$. The following conditions ensure correctness of isZero, when $\chi_{k}=D_{\mathbb{Z}, \sigma_{k}^{*}}$ (for all $\left.k \leq \kappa\right)$ : the first one implies that false negatives do not exist (if $u$ is level- $\kappa$ encoding of 0 , then isZero $(u)$ returns 1 ), whereas the second one implies that false positives occur with negligible probability.

$$
\begin{aligned}
& q>\max \left(\left(n \ell_{g^{-1}}\right)^{8},\left(\left(m_{r}+1\right) \cdot n \sigma_{1}^{*} \sigma^{\prime}\right)^{8 \kappa}\right) \\
& q>(2 n \sigma)^{4} .
\end{aligned}
$$

The aim of ext is to extract a quantity from its input $u=\left[c / z^{\kappa}\right]_{q}$ that depends only on the encoded value $[c]_{g}$, but not on the randomizers. To avoid trivial solutions, one requires that this extracted value has min-entropy $\geq 2 \lambda$ (if that is the 
- Instance generation InstGen $\left(1^{\lambda}, 1^{\kappa}\right)$ : Given security parameter $\lambda$ and multilinearity parameter $\kappa$, determine scheme parameters $n, q, m_{r}, \sigma, \sigma^{\prime}, \ell_{g^{-1}}, \ell$, based on the scheme analysis. Then proceed as follows:

- Sample $g \hookleftarrow D_{R, \sigma}$ until $\left\|g^{-1}\right\| \leq \ell_{g^{-1}}$ and $\mathcal{I}=\langle g\rangle$ is a prime ideal. Define encoding domain $R_{g}=R /\langle g\rangle$.

- Sample $z \hookleftarrow U\left(R_{q}\right)$.

- Sample a level-1 encoding of 1 : set $y=\left[a \cdot z^{-1}\right]_{q}$ with $a \hookleftarrow D_{1+I, \sigma^{\prime}}$.

- For $k \leq \kappa$, sample $m_{r}$ level- $k$ encodings of 0 : set $x_{j}^{(k)}=\left[b_{j}^{(k)} \cdot z^{-k}\right]_{q}$ with $b_{j}^{(k)} \hookleftarrow D_{I, \sigma^{\prime}}$ for all $j \leq m_{r}$.

(Note that $a=1+g r_{y}$ and $b_{j}^{(k)}=g r_{j}^{(k)}$ for some $r_{y}, r_{j}^{(k)} \in R$.)

- Sample $h \hookleftarrow D_{R, \sqrt{q}}$ and define the zero-testing parameter $p_{z t}=\left[\frac{h}{g} z^{\kappa}\right]_{q} \in R_{q}$.

- Return public parameters par $=\left(n, q, y,\left\{x_{j}^{(k)}\right\}_{j \leq m_{r}, k \leq \kappa}\right)$ and $p_{z t}$.

- Level-0 sampler samp(par): Sample $e \hookleftarrow D_{R, \sigma^{\prime}}$ and return $e$.

(Note that $e=e_{L}+g e_{H}$ for some unique coset representative $e_{L} \in \mathcal{P}_{g}$, and some $e_{H} \in R$.)

- Level- $k$ encoding enc $c_{k}$ (par, $e$ ): Given level-0 encoding $e \in R$ and parameters par:

- Encode $e$ at level $k$ : Compute $u^{\prime}=\left[e \cdot y^{k}\right]_{q}$.

- Re-randomize: Sample $\rho_{j} \hookleftarrow \chi_{k}$ for $j \leq m_{r}$ and return $u=\left[u^{\prime}+\sum_{j=1}^{m_{r}} \rho_{j} x_{j}^{(k)}\right]_{q}$. (Note that $u^{\prime}=\left[c^{\prime} / z^{k}\right]_{q}$ with $c^{\prime} \in e_{L}+I$ and $u=\left[\left(c^{\prime}+\sum_{j} \rho_{j} b_{j}^{(k)}\right) / z^{k}\right]_{q} \cdot$ )

- Adding encodings add: Given level- $k$ encodings $u_{1}=\left[c_{1} / z^{k}\right]_{q}$ and $u_{2}=\left[c_{2} / z^{k}\right]_{q}$ :

- Return $u=\left[u_{1}+u_{2}\right]_{q}$, a level- $k$ encoding of $\left[c_{1}+c_{2}\right]_{g}$.

- Multiplying encodings mult: Given level- $k_{1}$ encoding $u_{1}=\left[c_{1} / z^{k_{1}}\right]_{q}$ and a level$k_{2}$ encoding $u_{2}=\left[c_{2} / z^{k_{2}}\right]_{q}$ :

- Return $u=\left[u_{1} \cdot u_{2}\right]_{q}$, a level- $\left(k_{1}+k_{2}\right)$ encoding of $\left[c_{1} \cdot c_{2}\right]_{g}$.

- Zero testing at level $\kappa$ isZero(par, $\left.p_{z t}, u\right)$ : Given a level- $\kappa$ encoding $u=\left[c / z^{\kappa}\right]_{q}$, return 1 if $\left\|\left[p_{z t} u\right]_{q}\right\|_{\infty}<q^{3 / 4}$ and 0 else.

(Note that $\left[p_{z t} \cdot u\right]_{q}=[h c / g]_{q}$.)

- Extraction at level $\kappa \operatorname{ext}\left(\operatorname{par}, p_{z t}, u\right)$ : Given a level- $\kappa$ encoding $u=\left[c / z^{\kappa}\right]_{q}$, return $v=\operatorname{MSB}_{\ell}\left(\left[p_{z t} \cdot u\right]_{q}\right)$.

(Note that if $c=[c]_{g}+g r$ for some $r \in R$, then $v=M S B_{\ell}\left(\frac{h}{g}\left([c]_{g}+g r\right)\right)=$ $M S B_{\ell}\left(\frac{h}{g}[c]_{g}+h r\right)$, which is equal to $M S B_{\ell}\left(\frac{h}{g}[c]_{g}\right)$, with probability $1-\lambda^{-\omega(1)}$.)

Fig. 1. The GGH graded encoding scheme

case, then one can obtain a uniform distribution on $\{0,1\}^{\lambda}$, using a strong randomness extractor). The following two inequalities guarantee these properties, when $\chi_{k}=D_{\mathbb{Z}, \sigma_{k}^{*}}($ for all $k)$. The first one implies that $\varepsilon_{\text {ext }}=\operatorname{Pr}\left[\operatorname{ext}(u) \neq \operatorname{ext}\left(u^{\prime}\right)\right]$ is negligible, when $u$ and $u^{\prime}$ encode the same value $[c]_{g}$, whereas the second one provides large min-entropy.

$$
1 / 4 \log q-\log \left(\frac{2 n}{\varepsilon_{e x t}}\right) \geq \ell \geq \log \left(\frac{n \sigma}{8}\right) .
$$




\subsection{The GDDH, GCDH and Ext-GCDH Problems}

The computational problems that are required to be hard for the GGH scheme depend on the application. Here we recall the definitions of the Graded Decisional and Computational Diffie-Hellman (GDDH and GCDH) problems from [9]. We introduce another natural variant that we call the Extraction Graded Computational Diffie-Hellman (Ext-GCDH), in which the goal is to compute the extracted string of a Diffie-Hellman encoding.

Definition 3.1 (GCDH/Ext-GCDH/GDDH). The problems GCDH, ExtGCDH and GDDH are defined as follows with respect to experiment of Figure 21

- $\kappa$-graded $\boldsymbol{C D H}$ problem (GCDH): On inputs par, $p_{z t}$ and the $u_{i}$ 's of Step 2, output a level- $\kappa$ encoding of $\prod_{i \geq 0} e_{i}+\mathcal{I}$, i.e., $w \in R_{q}$ such that $\left\|\left[p_{z t}\left(v_{C}-w\right)\right]_{q}\right\| \leq q^{3 / 4}$.

- Extraction $\kappa$-graded CDH problem (Ext-GCDH): On inputs par, $p_{z t}$ and the $u_{i}$ 's of Step 2, output the extracted string for a level- $\kappa$ encoding of $\prod_{i \geq 0} e_{i}+\mathcal{I}$, i.e., $w=\operatorname{ext}\left(\operatorname{par}, p_{z t}, v_{C}\right)=M S B_{\ell}\left(\left[p_{z t} \cdot v_{C}\right]_{q}\right)$.

- $\kappa$-graded $\boldsymbol{D D H}$ problem (GDDH): Distinguish between $v_{D}$ and $v_{R}$, i.e., between the distributions $\mathcal{D}_{D D H}=\left\{\right.$ par, $\left.p_{z t},\left(u_{i}\right)_{0 \leq i \leq \kappa}, v_{D}\right\}$ and $\mathcal{D}_{R}=$ $\left\{\right.$ par, $\left.p_{z t},\left(u_{i}\right)_{0 \leq i \leq \kappa}, v_{R}\right\}$.

Given parameters $\lambda, n, q, m_{r}, \kappa, \sigma^{\prime}$, proceed as follows:

1. Run InstGen $\left(1^{n}, 1^{\kappa}\right)$ to get $\operatorname{par}=\left(n, q, y,\left\{x_{j}^{(k)}\right\}_{j, k}\right)$ and $p_{z t}$.

2. For $i=0, \ldots, \kappa$ :

-Sample $e_{i} \hookleftarrow D_{R, \sigma^{\prime}}, f_{i} \hookleftarrow D_{R, \sigma^{\prime}}$,

- Set $u_{i}=\left[e_{i} \cdot y+\sum_{j} \rho_{i j} x_{j}\right]_{q}$

with $\rho_{i j} \hookleftarrow \chi_{1}$ for all $j$.

3. Set $u^{*}=\left[\prod_{i=1}^{\kappa} u_{i}\right]_{q}$.

4. Set $v_{C}=\left[e_{0} u^{*}\right]_{q}$.

5. Sample $\rho_{j} \hookleftarrow \chi_{\kappa}$ for all $j$, set $v_{D}=\left[e_{0} u^{*}+\sum_{j} \rho_{j} x_{j}^{(\kappa)}\right]_{q}$.

6. Set $v_{R}=\left[f_{0} u^{*}+\sum_{j} \rho_{j} x_{j}^{(\kappa)}\right]_{q}$.
Given parameters $\lambda, n, q, m_{r}, \kappa,\left(\sigma_{k}^{*}\right)_{k \leq \kappa}$, proceed as follows:

1. Run InstGen $\left(1^{n}, 1^{\kappa}\right)$ to get par $=\left(n, q, y,\left\{x_{j}^{(k)}\right\}_{j, k}\right)$ and $p_{z t}$. Write $x_{j}^{(k)}=\left[b_{j}^{(k)} z^{-k}\right]_{q}$ and $B^{(k)}=\left[b_{1}^{(k)}, \cdots, b_{m_{r}}^{(k)}\right] \in \mathcal{I}^{m_{r}}$.

2. For $i=0, \ldots, \kappa$ : -Sample $e_{i} \hookleftarrow U\left(R_{g}\right), f_{i} \hookleftarrow U\left(R_{g}\right)$, -Set $u_{i}=\left[c_{i} z^{-1}\right]_{q} \hookleftarrow D_{\text {can }}^{(1)}\left(e_{i}\right)$ with $c_{i} \hookleftarrow D_{\mathcal{I}+e_{i}, \sigma_{1}^{*}\left(B^{(1)}\right)^{T}}$.

3. Set $u^{*}=\left[\prod_{i=1}^{\kappa} u_{i}\right]_{q}$.

4. Set $v_{C}=\left[e_{0} u^{*}\right]_{q}$.

5. Set $v_{D}=\left[c_{D} \cdot z^{-\kappa}\right]_{q} \hookleftarrow D_{c a n}^{(\kappa)}\left(\prod_{i=0}^{\kappa} e_{i}\right)$, with $c_{D} \hookleftarrow D_{\mathcal{I}+\prod_{i=0}^{\kappa} e_{i}, \sigma_{\kappa}^{*}\left(B^{(\kappa)}\right)^{T}}$.

6. Set $v_{R}=\left[c_{R} \cdot z^{-\kappa}\right]_{q} \hookleftarrow D_{\text {can }}^{(\kappa)}\left(f_{0} \prod_{i=1}^{\kappa} e_{i}\right)$, with $c_{R} \hookleftarrow D_{\mathcal{I}+f_{0}} \prod_{i=1}^{\kappa} e_{i}, \sigma_{\kappa}^{*}\left(B^{(\kappa)}\right)^{T}$.

Fig. 2. The GGH security experiment

Fig. 3. The canonical security experiment

${ }^{1}$ Note that we use a slightly different process from [9], by adding a re-randomization to the element $v_{D}$. Without it, there exists a "division attack" against GDDH. 
Ext-GCDH is at least as hard as GDDH: given $v_{x}$ with $x \in\{\mathrm{DDH}, \mathrm{R}\}$, use the Ext-GCDH oracle to compute $w=\operatorname{ext}\left(\operatorname{par}, p_{z t}, v_{C}\right)$. Nevertheless, we show (see full version) that it suffices for instantiating, in the random oracle model, at least some of the interesting applications of graded encoding schemes, at a higher efficiency than the instantiations of [9] based on GDDH.

\subsection{The GGH Re-randomization Security Requirement}

The encoding re-randomization step in the GGH scheme is necessary for the hardness of the problems above. In [9], Garg et al. imposed the informal requirement that the re-randomization process "erases" the structure of the input encoding, while preserving the encoded coset. In setting parameters, they interpreted this requirement in the following natural way.

Definition 3.2 (Strong re-randomization security requirement). Let $u^{\prime}=$ $\left[c^{\prime} / z^{k}\right]_{q}$, with $c^{\prime}=e_{L}+g r^{\prime}$ be a fixed level- $k$ encoding of $e_{L} \in R_{g}$, and let $u=\left[u^{\prime}+\sum_{j} \rho_{j} x_{k}^{(j)}\right]_{q}=\left[c / z^{k}\right]_{q}$ with $c=e_{L}+g r$ and $r=r^{\prime}+\sum_{j} \rho_{j} r_{j}^{(k)}$ be the re-randomized encoding, with $\rho_{j} \hookleftarrow \chi_{k}$ for $j \leq m_{r}$. Let $D_{u}^{(k)}\left(e_{L}, r^{\prime}\right)$ denote the distribution of $u$ (over the randomness of $\rho_{j}$ 's), parameterized by $\left(e_{L}, r^{\prime}\right)$ and let $D_{\mathrm{can}}^{(k)}\left(e_{L}\right)$ denote some canonical distribution, parameterized by $e_{L}$, that is independent of $r^{\prime}$. Then we say that the strong re-randomization security requirement is satisfied at level $k$ with respect to $D_{\text {can }}^{(k)}\left(e_{L}\right)$ and encoding norm $\gamma^{(k)}$ if $\Delta\left(D_{u}^{(k)}\left(e_{L}, r^{\prime}\right), D_{\text {can }}^{(k)}\left(e_{L}\right)\right) \leq 2^{-\lambda}$ for any $u^{\prime}=\left[c^{\prime} / z^{k}\right]_{q}$ with $\left\|c^{\prime}\right\| \leq \gamma^{(k)}$.

The authors of [9] argued that with $\chi_{k}=D_{\mathbb{Z}, \sigma_{k}^{*}}($ for $k \leq \kappa)$ and a "drowning ratio" $\sigma_{k}^{*} /\left\|r^{\prime}\right\|$ exponential in security parameter $\lambda$, the distribution $D_{u}^{(k)}\left(e_{L}, r^{\prime}\right)$ is within negligible statistical distance to the canonical distribution $D_{\text {can }}^{(k)}\left(e_{L}\right)=$ $\left[D_{\mathcal{I}+e_{L}, \sigma_{k}^{*}\left(B^{(k)}\right)^{T}} \cdot z^{-k}\right]_{q}$. This requirement may be stronger than needed. Accordingly, we now clarify the desired goal.

\subsection{Our Security Goal: Canonical Assumptions}

We formalize a re-randomization security goal to capture a security guarantee against "statistical correlation" attacks on GCDH/Ext-GCDH/GDDH. We define canonical variants cGCDH/Ext-cGCDH/cGDDH of GCDH/ExtGCDH/GDDH, using Figure 3. The main difference with Figure 2 is that the encodings $u_{i}=\left[c_{i} / z\right]_{q}$ of the hidden elements $e_{i}$, are sampled from a canonical distribution $D_{\text {can }}^{(1)}\left(e_{i}\right)$, parameterized by $e_{i}$, whose statistical parameters are independent of the encoded coset $e_{i}$, so that it is "by construction" immune against statistical correlation attacks. In particular, in the canonical distribution $D_{\text {can }}^{(1)}\left(e_{i}\right)$ that we use, $c_{i}$ is sampled from a discrete Gaussian distribution $D_{\mathcal{I}+e_{i}, \sigma_{1}^{*}\left(B^{(1)}\right)^{T}}$ (over the choice of the randomization, for a fixed $e_{i}$ ), whose statistical parameters such as center (namely 0 ) and deviation matrix $\sigma_{1}^{*}\left(B^{(1)}\right)^{T}$ are independent of $e_{i}$. The only dependence this distribution has on the encoded element $e_{i}$ is via its support $\mathcal{I}+e_{i}$. 
We believe the canonical problems are cleaner and more natural than the non-canonical variants, since they decouple the re-randomization aspect from the rest of the computational problem. As a further simplification, the canonical variants also have their level-0 elements $e_{i}$ distributed uniformly on $R_{g}$ (rather than as reductions mod $\mathcal{I}$ of Gaussian samples).

Definition 3.3 (cGCDH/Ext-cGCDH/cGDDH). The canonical problems $c G C D H$, Ext-cGCDH and $c G D D H$ are defined as follows with respect to the experiment of Figure 3 and canonical encoding distribution $D_{c a n}^{(k)}(e)$ (parameterized by encoding level $k$ and encoded element $e)$ :

- cGCDH: On inputs par, $p_{z t}$ and the $u_{i}$ 's, output $w \in R_{q}$ such that $\left\|\left[p_{z t}\left(v_{C}-w\right)\right]_{q}\right\| \leq q^{3 / 4}$.

- Ext-cGCDH: On inputs par, $p_{z t}$ and the $u_{i}$ 's, output: $w=\operatorname{ext}\left(\operatorname{par}, p_{z t}, v_{C}\right)=M S B_{\ell}\left(\left[p_{z t} \cdot v_{C}\right]_{q}\right)$.

- $\boldsymbol{c G D D H : ~ D i s t i n g u i s h ~ b e t w e e n ~} \mathcal{D}_{D D H}=\left\{\right.$ par, $\left.p_{z t},\left(u_{i}\right)_{0 \leq i \leq \kappa}, v_{D}\right\}$ and $\mathcal{D}_{R}=$ $\left\{\right.$ par, $\left.p_{z t},\left(u_{i}\right)_{0 \leq i \leq \kappa}, v_{R}\right\}$.

REMARK. One could consider alternative definitions of natural canonical encoding distributions besides the one we adopt here (see full paper for examples for which our results also apply).

Given the canonical problems on whose hardness we wish to rely, our security goal for re-randomization with respect to the GCDH (resp. Ext-GCDH/GDDH) problems can now be easily formulated: hardness of the latter should be implied by hardness of the former.

Definition 3.4 (Re-randomization security goal). We say that the rerandomization security goal is satisfied with respect to GCDH (resp. Ext$G C D H / G D D H)$ if any adversary against GCDH (resp. Ext-GCDH/ GDDH) with run-time $T=O\left(2^{\lambda}\right)$ and advantage $\varepsilon=\Omega\left(2^{-\lambda}\right)$ can be used to construct an adversary against $c G C D H$ (resp. Ext-cGCDH/cGDDH) with run-time $T^{\prime}=\operatorname{poly}(T, \lambda)$ and advantage $\varepsilon^{\prime}=\Omega(\operatorname{poly}(\varepsilon, \lambda))$.

\section{Polynomial Drowning via Rényi Divergence}

In this section, we present our first result towards our improvement of the GGH scheme re-randomization. It shows that one may reduce the re-randomization "drowning" ratio $\sigma_{k}^{*} /\left\|r^{\prime}\right\|$ from exponential to polynomial in the security parameter $\lambda$. Although the SD between the re-randomized encoding distribution $D_{1}$ (essentially a discrete Gaussian with an added offset vector $r^{\prime}$ ) and the desired canonical encoding distribution $D_{2}$ (a discrete Gaussian without an added offset vector) is then non-negligible, we show that these encoding distributions are still sufficiently close with respect to an alternative closeness measure to the SD, in the sense that switching between them preserves the success probability of any search problem adversary receiving these encodings as input, up to a small multiplicative constant. This allows us to show that our re-randomization goal is satisfied for the search problems GCDH and Ext-GCDH. 
Technically, the closeness measure we study is the Rényi divergence $R\left(D_{1} \| D_{2}\right)$ between the distributions $D_{1}$ and $D_{2}$, defined as the expected value of $D_{1}(r) / D_{2}(r)$ over the randomness of $r$ sampled from $D_{1}$ (for brevity we will call $R\left(D_{1} \| D_{2}\right)$ the RD between $D_{1}$ and $D_{2}$ ). Intuitively, the RD is an alternative to SD as measure of distribution closeness, where we replace the difference between the distributions in $\mathrm{SD}$, by the ratio of the distributions in RD. Accordingly, one may hope RD to have analogous properties to SD, where addition in the property of SD is replaced by multiplication in the analogous property of RD. Remarkably, this holds true in some sense, and we explore some of this below. In particular, a very important property of the SD is that for any two distributions $D_{1}, D_{2}$ on space $X$, and any event $E \subseteq X$, we have $D_{1}(E) \geq D_{2}(E)-\Delta\left(D_{1}, D_{2}\right)$. Lyubashevsky et al. [13] observed an analogous property of the RD that follows roughly the above intuition: $D_{1}(E) \geq D_{2}(E)^{2} / R\left(D_{1} \| D_{2}\right)$. The latter property implies that as long as $R\left(D_{1} \| D_{2}\right)$ is bounded as poly $(\lambda)$, any event of non-negligible probability $D_{2}(E)$ under $D_{2}$ will also have non-negligible probability $D_{1}(E)$ under $D_{1}$. We show that for our offset discrete Gaussian distributions $D_{1}, D_{2}$ above, we have $R\left(D_{1} \| D_{2}\right)=$ $O(\operatorname{poly}(\lambda))$, if $\sigma_{k}^{*} /\left\|r^{\prime}\right\|=\Omega(\operatorname{poly}(\lambda))$, as required for our re-randomization security goal.

The Rényi divergence $(R D)$ and its properties. We review the $\mathrm{RD}$ [18] and some of its properties. For convenience, our definition of the RD is the exponential of the usual definition used in information theory [8], and coincides with a discrete version of the quantity $R$ defined for continuous density functions in 13. Claim 5.11].

For any two discrete probability distributions $P$ and $Q$ such that $\operatorname{Supp}(P) \subseteq$ $\operatorname{Supp}(Q)$ over a domain $X$ and $\alpha>1$, we define the Rényi Divergence of orders $\alpha$ and $\infty$ by

$$
R_{\alpha}(P \| Q)=\left(\sum_{x \in X} \frac{P(x)^{\alpha}}{Q(x)^{\alpha-1}}\right)^{\frac{1}{\alpha-1}} \text { and } R_{\infty}(P \| Q)=\max _{x \in X} \frac{P(x)}{Q(x)}
$$

with the convention that the fraction is zero when both numerator and denominator are zero. A convenient choice for computations (as also used in [13]) is $\alpha=2$, in which case we omit $\alpha$. Note that $R_{\alpha}(P \| Q)^{\alpha-1}=\sum_{x} P(x) \cdot(P(x) / Q(x))^{\alpha-1} \leq$ $R_{\infty}(P \| Q)^{\alpha-1}$. We list several properties of the RD that can be considered the multiplicative analogues of those of the SD. The following lemma is proven in the full version.

Lemma 4.1. Let $P_{1}, P_{2}, P_{3}$ and $Q_{1}, Q_{2}, Q_{3}$ denote discrete distributions on a domain $X$ and let $\alpha \in(1, \infty]$. Then the following properties hold:

- Log. Positivity: $R_{\alpha}\left(P_{1} \| Q_{1}\right) \geq R_{\alpha}\left(P_{1} \| P_{1}\right)=1$.

- Data Processing Inequality: $R_{\alpha}\left(P_{1}^{f} \| Q_{1}^{f}\right) \leq R_{\alpha}\left(P_{1} \| Q_{1}\right)$ for any function $f$, where $P_{1}^{f}$ (resp. $Q_{1}^{f}$ ) denotes the distribution of $f(y)$ induced by sampling $y \hookleftarrow P_{1}$ (resp. $y \hookleftarrow Q_{1}$ ).

- Multiplicativity: Let $P$ and $Q$ denote any two distributions of a pair of random variables $\left(Y_{1}, Y_{2}\right)$ on $X \times X$. For $i \in\{1,2\}$, assume $P_{i}$ (resp. $\left.Q_{i}\right)$ is the marginal distribution of $Y_{i}$ under $P$ (resp. Q), and let $P_{2 \mid 1}\left(\cdot \mid y_{1}\right)$ (resp. 
$\left.Q_{2 \mid 1}\left(\cdot \mid y_{1}\right)\right)$ denote the conditional distribution of $Y_{2}$ given that $Y_{1}=y_{1}$. Then we have:

- $R_{\alpha}(P \| Q)=R_{\alpha}\left(P_{1} \| Q_{1}\right) \cdot R_{\alpha}\left(P_{2} \| Q_{2}\right)$ if $Y_{1}$ and $Y_{2}$ are independent.

- $R_{\alpha}(P \| Q) \leq R_{\infty}\left(P_{1} \| Q_{1}\right) \cdot \max _{y_{1} \in X} R_{\alpha}\left(P_{2 \mid 1}\left(\cdot \mid y_{1}\right) \| Q_{2 \mid 1}\left(\cdot \mid y_{1}\right)\right)$.

- Weak Triangle Inequality: We have:

$$
R_{\alpha}\left(P_{1} \| P_{3}\right) \leq\left\{\begin{array}{r}
R_{\alpha}\left(P_{1} \| P_{2}\right) \cdot R_{\infty}\left(P_{2} \| P_{3}\right), \\
R_{\infty}\left(P_{1} \| P_{2}\right)^{\frac{\alpha}{\alpha-1}} \cdot R_{\alpha}\left(P_{2} \| P_{3}\right) .
\end{array}\right.
$$

- $R_{\infty}$ Triangle Inequality: If $R_{\infty}\left(P_{1} \| P_{2}\right)$ and $R_{\infty}\left(P_{2} \| P_{3}\right)$ are defined, then $R_{\infty}\left(P_{1} \| P_{3}\right) \leq R_{\infty}\left(P_{1} \| P_{2}\right) \cdot R_{\infty}\left(P_{2} \| P_{3}\right)$.

- Probability Preservation: Let $A \subseteq X$ be an arbitrary event. Then $Q_{1}(A) \geq$ $P_{1}(A)^{\frac{\alpha}{\alpha-1}} / R_{\alpha}\left(P_{1} \| Q_{1}\right)$.

We note that the RD does not satisfy the (multiplicative) triangle inequality $R\left(P_{1} \| P_{3}\right) \leq R\left(P_{1} \| P_{2}\right) \cdot R\left(P_{2} \| P_{3}\right)$ in general (see [8]), but a weaker inequality holds if one of the pairs of distributions has a bounded $R_{\infty}$ divergence, as shown above. We also observe that $R_{\infty}$ does satisfy the triangle inequality.

For our re-randomization application, we are interested in the RD between two discrete Gaussians with the same deviation matrix $S$, that differ by some fixed offset vector $d$. The following result (proved in the full version) shows that their RD is $O(1)$ if $\sigma_{n}(S) /\|d\|=\Omega(1)$.

Lemma 4.2. For any $n$-dimensional lattice $\Lambda$ in $\mathbb{R}^{n}$ and matrix $S$, let $P$ be the distribution $D_{\Lambda, S, w}$ and $Q$ be the distribution $D_{\Lambda, S, z}$ for some fixed $w, z \in \mathbb{R}^{n}$. If $w, z \in \Lambda$, let $\varepsilon=0$. Otherwise, fix $\varepsilon \in(0,1)$ and assume that $\sigma_{n}(S) \geq \eta_{\varepsilon}(\Lambda)$. Then $R(P \| Q) \leq\left(\frac{1+\varepsilon}{1-\varepsilon}\right)^{2} \cdot \exp \left(2 \pi\|w-z\|^{2} / \sigma_{n}(S)^{2}\right)$.

\section{A Discrete Gaussian Leftover Hash Lemma over $\boldsymbol{R}$}

In this section, we present our second main result for improving the GGH scheme re-randomization algorithm. Recall that the GGH algorithm re-randomizes a level- $k$ encoding $u^{\prime}$ into $u=\left[u^{\prime}+\sum_{j=1}^{m_{r}} \rho_{j} x_{j}^{(k)}\right]_{q}$, where the $\rho_{j}$ 's are sampled from $\chi_{1}=D_{\mathbb{Z}, \sigma_{1}^{*}}$ and $x_{j}^{(k)}=\left[b_{j}^{(k)} / z^{k}\right]_{q}=\left[g r_{j}^{(k)} / z^{k}\right]_{q}$. To show that the distribution of $\sum_{j=1}^{m_{r}} \rho_{j} b_{j}^{(k)}$ is close to a discrete Gaussian over $\mathcal{I}$, they then apply the discrete Gaussian LHL from [1, Th. 3], using $m_{r}=\Omega(n \log n)$ fixed elements $b_{j}^{(k)} \in \mathcal{I}$ that are published obliviously as randomizers "inside" the public zeroencodings $x_{j}^{(k)}$. We show that it suffices to sample 2 randomizers as elements of the full $n$-dimensional ring $R$, rather than just from $\mathbb{Z}$, i.e., we set $\chi_{1}=D_{R, \sigma_{1}^{*}}$. Our proof follows the same high-level steps as the proof of [1, Th. 3], but differs technically, as explained in the introduction.

For a fixed $X=\left(x_{1}, x_{2}\right) \in R^{2}$, we define the distribution $\widetilde{\mathcal{E}}_{X, s}=x_{1} D_{R, s}+$ $x_{2} D_{R, s}$ as the distribution induced by sampling $\boldsymbol{u}=\left(u_{1}, u_{2}\right) \in R^{2}$ from a discrete spherical Gaussian with parameter $s$, and outputting $y=x_{1} u_{1}+x_{2} u_{2}$. We prove the following result on $\widetilde{\mathcal{E}}_{X, s}$. 
Theorem 5.1. Let $R=\mathbb{Z}[x] /\left\langle x^{n}+1\right\rangle$ with $n$ a power of 2 and $\mathcal{I}=\langle g\rangle \subseteq R$, for some $g \in R$. Fix $\varepsilon \in(0,1 / 3), X=\left(x_{1}, x_{2}\right) \in \mathcal{I}^{2}$ and $s>0$ satisfying the conditions

- Column span: $X \cdot R^{2}=\mathcal{I}$.

- Smoothing: $s \geq \max \left(\left\|g^{-1} x_{1}\right\|_{\infty},\left\|g^{-1} x_{2}\right\|_{\infty}\right) \cdot n \cdot \sqrt{\frac{2}{\pi} \log (2 n(1+1 / \varepsilon))}$.

Then, for all $x \in \mathcal{I}$ we have $\widetilde{\mathcal{E}}_{X, s}(x) \in\left[\frac{1-\varepsilon}{1+\varepsilon}, 1\right] \cdot D_{\mathcal{I}, s X^{T}}(x)$. In particular, we have $\Delta\left(\widetilde{\mathcal{E}}_{X, s}, D_{\mathcal{I}, s X^{T}}\right) \leq 2 \varepsilon$. Finally, if $s \cdot \sigma_{n}\left(g^{-1}\right) \geq 7 n^{1.5} \ln ^{1.5}(n) .2 x_{1}, x_{2} \hookleftarrow D_{\mathcal{I}, s}$ and $n$ grows to infinity, then the first condition holds with probability $\Omega(1)$.

We prove this result for $g=1$, and then we generalize to general $g$. First, we consider the column span condition.

Lemma 5.2 (Adapted from [23, Le. 4.2 and Le. 4.4]). Let $S \in \mathbb{R}^{n \times n}$, and $\sigma_{n}(S) \geq 7 n^{1.5} \ln ^{1.5}(n)$. For $n$ going to infinity, we have $\operatorname{Pr}_{x_{1}, x_{2} \hookleftarrow D_{R, S}}\left[X \cdot R^{2}=\right.$ $R] \geq \Omega(1)$.

Let $A_{X} \subseteq\left\{\left(v_{1}, v_{2}\right) \in R^{2}: x_{1} v_{1}+x_{2} v_{2}=0\right\}$ be the 1-dimensional $R$-module of vectors orthogonal to $X$. We view $A_{X}$ as an $n$-dimensional lattice in $\mathbb{Z}^{2 n}$, via the polynomial-to-coefficient-vector mapping.

Lemma 5.3 (Adapted from [1, Le. 10]). Fix $X$ such that $X \cdot R^{2}=R$ and $A_{X}$ as above. If $s \geq \eta_{\varepsilon}\left(A_{X}\right)$, then $\widetilde{\mathcal{E}}_{X, s}(z) \in\left[\frac{1-\varepsilon}{1+\varepsilon}, 1\right] \cdot D_{\mathbb{Z}^{n}, s X^{T}}(z)$ for any $z \in R$.

We now study the quantity $\eta_{\varepsilon}\left(A_{X}\right)$. First, we show that all successive Minkowski minima of $A_{X}$ are equal. This property is inherited from the "equal minima property" of ideal lattices in $R$.

Lemma 5.4. Let $X$ and $A_{X}$ be as above. Then $\lambda_{1}\left(A_{X}\right)=\cdots=\lambda_{n}\left(A_{X}\right)$.

Lemma 5.5. Let $X$ and $A_{X}$ be as above. Let $s \geq \max \left(\left\|x_{1}\right\|_{\infty},\left\|x_{2}\right\|_{\infty}\right)$. Then we have: $\eta_{\varepsilon}\left(A_{X}\right) \leq s n \cdot \sqrt{\frac{2}{\pi} \log (2 n(1+1 / \varepsilon))}$.

Combining the above lemmas, we get Theorem 5.1 for $g=1$. The general case is proved as follows. The injective map $y \mapsto g \cdot y$ on $R$ takes the distribution $\widetilde{\mathcal{E}}_{\bar{X}, s}$ with $\bar{X}=g^{-1} \cdot X$ to the distribution $\widetilde{\mathcal{E}}_{X, s}$, while it takes $D_{R, s \bar{X}^{T}}$ to $D_{\mathcal{I}, s X^{T}}$, with $I=\langle g\rangle$. The conditions $X \cdot R^{2}=\mathcal{I}$ and $\bar{X} \cdot R^{2}=R$ are equivalent. The smoothing condition is satisfied for $\bar{X}$ by the choice of $s$. Thus we can apply Theorem [5.1] with $g=1$ to $\widetilde{\mathcal{E}}_{\bar{X}, s}$, and conclude by applying the mapping $M_{g}$ to get the general case of Theorem [5.1] For the very last statement of Theorem 5.1] it suffices to observe that $D_{\mathcal{I}, s}=g \cdot D_{R, s\left(g^{-1}\right)^{T}} 3$

\footnotetext{
${ }^{2}$ By abuse of notation, we identify $g^{-1} \in K$ with the linear map over $\mathbb{Q}^{n}$ obtained by applying the polynomial-to-coefficient-vector mapping to the map $r \mapsto g^{-1} r$.

${ }^{3}$ With the same abuse of notation as in the previous footnote, for the term $\left(g^{-1}\right)^{T}$.
} 


\section{Our Improved GGH Grading Scheme: GGHLite}

We are now ready to describe our simpler and more efficient variant of the GGH grading scheme, that we call GGHLite. The scheme is summarized in Figure 4 The modifications from the original GGH scheme consist in:

- Using $m_{r}=2$ re-randomization elements $x_{1}, x_{2}$ in the public key, sampling the randomizers $\rho_{1}, \rho_{2}$ from a discrete Gaussian $D_{R, \sigma_{1}^{*}}$ over the whole ring $R$ (rather than from $\mathbb{Z}$ ), applying our algebraic ring variant of the LHL from Section 5 .

- Saving an exponential factor $\approx 2^{\lambda}$ in the re-randomization parameter $\sigma_{1}^{*}$ by applying the RD bounds from Section 4

In terms of re-randomization security requirement, we relax the strong SDbased requirement on the original GGH scheme to the following weaker RD-based requirement on GGHLite.

Definition 6.1 (Weak re-randomization security requirement). Using the notations of Definition 3.2, we say that the weak re-randomization security requirement is satisfied at level $k$ with respect to $D_{\mathrm{can}}^{(k)}\left(e_{L}\right)$ and encoding norm $\gamma^{(k)}$ if $R\left(D_{u}^{(k)}\left(e_{L}, r^{\prime}\right) \| D_{\text {can }}^{(k)}\left(e_{L}\right)\right)=O(\operatorname{poly}(\lambda))$ for any $u^{\prime}=\left[c^{\prime} / z^{k}\right]_{q}$ such that $\left\|c^{\prime}\right\| \leq \gamma^{(k)}$.

We summarize GGHLite in Figure 4, which only shows the algorithms differing from those in the GGH scheme of Figure 1

- Instance generation InstGen $\left(1^{\lambda}, 1^{\kappa}\right)$ : Given security parameter $\lambda$ and multilinearity parameter $\kappa$, determine scheme parameters $n, q, m_{r}=2, \sigma, \sigma^{\prime}, \ell_{g^{-1}}, \ell_{b}, \ell$, based on the scheme analysis. Then proceed as follows:

- Sample $g \hookleftarrow D_{R, \sigma}$ until $\left\|g^{-1}\right\| \leq \ell_{g^{-1}}$ and $\mathcal{I}=\langle g\rangle$ is a prime ideal.

- Sample $z \hookleftarrow U\left(R_{q}\right)$.

- Sample a level-1 encoding of 1: $y=\left[a \cdot z^{-1}\right]_{q}$ with $a \hookleftarrow D_{1+I, \sigma^{\prime}}$.

- For $k \leq \kappa$ :

* Sample $B^{(k)}=\left(b_{1}^{(k)}, b_{2}^{(k)}\right)$ from $\left(D_{I, \sigma^{\prime}}\right)^{2}$. If $\left\langle b_{1}^{(k)}, b_{2}^{(k)}\right\rangle \neq \mathcal{I}$, or $\sigma_{n}\left(\operatorname{rot}\left(B^{(k)}\right)\right)<\ell_{b}$, then re-sample.

* Define level- $k$ encodings of $0: x_{1}^{(k)}=\left[b_{1}^{(k)} \cdot z^{-k}\right]_{q}, x_{2}^{(k)}=\left[b_{2}^{(k)} \cdot z^{-k}\right]_{q}$.

- Sample $h \hookleftarrow D_{R, \sqrt{q}}$ and define the zero-testing parameter $p_{z t}=\left[\frac{h}{g} z^{\kappa}\right]_{q} \in R_{q}$.

- Return public parameters par $=\left(n, q, y,\left\{\left(x_{1}^{(k)}, x_{2}^{(k)}\right)\right\}_{k \leq \kappa}\right)$ and $p_{z t}$.

- Level- $k$ encoding enc en (par, $e)$ : Given level-0 encoding $e \in R$ and parameters par:

- Encode $e$ at level $k$ : Compute $u^{\prime}=\left[e \cdot y^{k}\right]_{q}$.

- Return $u=\left[\left(u^{\prime}+\rho_{1} \cdot x_{1}^{(k)}+\rho_{2} \cdot x_{2}^{(k)}\right)\right]_{q}$, with $\rho_{1}, \rho_{2} \hookleftarrow D_{R, \sigma_{k}^{*}}$.

Fig. 4. The new algorithms of our GGHLite scheme 
Choice of $\sigma, \ell_{g^{-1}}$ and $\sigma^{\prime}, \ell_{b}$. The upper bound $\ell_{g^{-1}}$ on $\left\|g^{-1}\right\|$ in the rejection test of InstGen can be chosen as small as possible while keeping the rejection probability $p_{g}$ bounded from 1 . According to Lemma 2.3 with $t=2 \sqrt{2 \pi \mathrm{e}} n p_{g}^{-1}$ and $\delta=1 / 3$, one can choose

$$
\ell_{g^{-1}}=4 \sqrt{\pi \mathrm{e} n} /\left(p_{g} \sigma\right) \text { and } \sigma \geq 2 n \sqrt{\mathrm{e} \ln (8 n) / \pi} / p_{g},
$$

to achieve $p_{g}<1$. Note that the same choices apply to the GGH scheme: here we have a rigorous bound on $p_{g}$ instead of the heuristic arguments for estimating in $\left\|g^{-1}\right\|$ in $[9$; however, as in [9], we do not have a rigorous bound on the probability that $\mathcal{I}$ is prime conditioned on this choice.

Let $p_{b}$ be the rejection probability for the lower bound $\ell_{b}$ on $\sigma_{n}\left(B^{(k)}\right)$ in the rejection test of InstGen. To keep $p_{b}$ away from 1 , we use that $\sigma_{n}\left(B^{(k)}\right)^{2}=$ $\min _{u \in K,\|u\|=1} \sum_{i=1,2}\left\|u \cdot b_{i}^{(k)}\right\|^{2} \geq \sum_{i=1,2} \sigma_{n}\left(b_{i}^{(k)}\right)^{2}$. Applying Lemma 2.3 with $t=2 \sqrt{2 \pi \mathrm{e}} n p_{b}^{-1}$ and $\delta=1 / 3$, we get that $\sigma_{n}\left(b_{i}^{(k)}\right)>\frac{p_{b}}{8 \sqrt{\pi \mathrm{e} n}} \cdot \sigma^{\prime}$, except with probability $\leq p_{b}$ for $i \in\{1,2\}$ if $\sigma^{\prime} \geq \frac{t}{\sqrt{2 \pi}} \eta_{1 / 3}(\mathcal{I})$, where $\eta_{1 / 3}(\mathcal{I}) \leq \sqrt{\ln (8 n) / \pi}$. $\|g\|$ by Lemma 2.1. Therefore, we can choose

$$
\ell_{b}=\frac{p_{b}}{2 \sqrt{\pi \mathrm{e} n}} \cdot \sigma^{\prime} \text { and } \sigma^{\prime} \geq 2 n^{1.5} \sigma \sqrt{\mathrm{e} \ln (8 n) / \pi} / p_{b}
$$

Zero-testing and extraction correctness. The correctness conditions for zerotesting and correctness remain the same as conditions (2), (3) for the original GGH scheme. The only modification needed is for condition (11), because in GGHLite, $m_{r}=2$ and $\rho_{j} \in R$ so $\left\|\rho_{j} b_{j}^{(1)}\right\| \leq \sqrt{n}\left\|\rho_{j}\right\|\left\|b_{j}^{(1)}\right\|$. Accordingly, condition (1) is replaced by:

$$
q>\max \left(\left(n \ell_{g^{-1}}\right)^{8},\left(3 \cdot n^{1.5} \sigma^{*} \sigma^{\prime}\right)^{8 \kappa}\right) .
$$

Security. We state our improved re-randomization security reduction for GGHLite, that works with much smaller parameters than GGH. To our knowledge, it is the first security proof in which the RD is used to replace the SD in a sequence of games, using the RD properties from Section 4 to combine the bounds on changes between games. This allows us to gain the benefits of RD over SD, for both the drowning and smoothing aspects. Namely, with $\varepsilon_{d}, \varepsilon_{\rho}, \varepsilon_{e}$ in Theorem 6.2 set as large as $O(\log \lambda / \kappa)$, our weak security requirement of Definition 6.1] is satisfied (the RD between real and canonical encoding distributions is bounded by the quantity $R=\operatorname{poly}(\lambda)$ in Theorem 6.2), and our re-randomization goal for Ext$\mathrm{GCDH}$ is achieved (whereas the strong requirement of Definition 3.2 is not satisfied).

Theorem 6.2 (Security of GGHLite). Let $\varepsilon_{d}, \varepsilon_{\rho}, \varepsilon_{e} \in(0,1 / 2)$ and $\kappa \leq 2^{n}$. Suppose that the following conditions are satisfied for GGHLite:

\section{- LHL Smoothing:}

$$
\sigma_{1}^{*} \geq n^{1.5} \cdot \ell_{g^{-1}} \cdot \sigma \cdot \sqrt{2 \log \left(4 n \cdot \varepsilon_{\rho}^{-1}\right) / \pi} .
$$


- Offset "Drowning:"

$$
\sigma_{1}^{*} \geq n^{1.5} \cdot\left(\sigma^{\prime}\right)^{2} \cdot \sqrt{2 \pi \varepsilon_{d}^{-1}} / \ell_{b}
$$

- samp Uniformity Smoothing:

$$
\sigma^{\prime} \geq \sigma \cdot \sqrt{n \ln \left(4 n \cdot \varepsilon_{e}^{-1}\right) / \pi}
$$

Then, if $A$ is an adversary against the (non-canonical) Ext-GCDH problem for GGHLite with run-time $T$ and advantage $\varepsilon$, then $A$ is also an adversary against the canonical problem Ext-cGCDH for GGHLite with $T^{\prime}=T$ and advantage

$$
\varepsilon^{\prime} \geq\left(\varepsilon-O\left(\kappa \cdot 2^{-n}\right)\right)^{2} / R \text { with } R=2^{O\left(\kappa \cdot\left(\varepsilon_{d}+\varepsilon_{\rho}+\varepsilon_{e}+2^{-n}\right)\right)} .
$$

In particular, there exist $\varepsilon_{d}, \varepsilon_{e}, \varepsilon_{\rho}$ bounded as $O(\log \lambda / \kappa)$ such that the rerandomization security goal in Definition 3.4 is satisfied by GGHLite with respect to problem Ext-GCDH.

\section{Parameter Settings}

In Table 11 we summarize asymptotic parameters for GGHLite to achieve $2^{\lambda}$ security for the underlying Ext-GCDH problem, assuming the hardness of the canonical Ext-cGCDH problem, and to satisfy the zero-testing/extraction correctness conditions with error probability $\lambda^{-\omega(1)}$. For simplicity, we assume that $\kappa=\omega(1)$. For comparison, we also show the corresponding parameters for $\mathrm{GGH}$. The "Condition" column lists the conditions that determine the corresponding parameter in the case of GGHLite. For security of the canonical Ext-cGCDH problem, we assume (as in 9]) that the best attack is the one described in [9. Se. 6.3.3], whose complexity is dominated by the cost of solving $\gamma$-SVP (the

Table 1. Asymptotic parameters

\begin{tabular}{cccc}
\hline \hline Parameter & GGHLite & GGH[9] & Condition \\
\hline$m_{r}$ & 2 & $\Omega(n \log n)$ & LHL: Th. [5.1] \\
$\sigma$ & $O(n \log n)$ & $O(n \log n)$ & Eq. (4) \\
$\ell_{g^{-1}}$ & $O(1 / \sqrt{n \log n})$ & $O(1 / \sqrt{n \log n})$ & Eq. (41) \\
$\varepsilon_{d}, \varepsilon_{e}, \varepsilon_{\rho}$ & $O\left(\kappa^{-1}\right)$ & $O\left(2^{-\lambda} \kappa^{-1}\right)$ & Eq. (10) \\
$\sigma^{\prime}$ & $\widetilde{O}\left(n^{2.5}\right)$ & $\widetilde{O}\left(n^{1.5} \sqrt{\lambda}\right)$ & Eq. (5) \\
$\sigma_{1}^{*}$ & $\widetilde{O}\left(n^{4.5} \sqrt{\log \kappa}\right)$ & $\widetilde{O}\left(2^{\lambda} n^{4.5}(\lambda+\log \kappa)\right)$ & Drown: Eq. (8) \\
$\varepsilon_{\text {ext }}$ & $O\left(\lambda^{-\omega(1)}\right)$ & $O\left(\lambda^{-\omega(1)}\right)$ & \\
$q$ & $\widetilde{O}\left(\left(n^{8.5} \sqrt{\log \kappa}\right)^{8 \kappa}\right)$ & $\widetilde{O}\left(\left(2^{\lambda} n^{8} \lambda^{1.5}\right)^{8 \kappa}\right)$ & Corr.: Eq. (6) \\
$n$ & $O(\kappa \lambda \log \lambda)$ & $O\left(\kappa \lambda^{2}\right)$ & SVP: Eq. (11) \\
$\mid$ enc| & $O\left(\kappa^{2} \lambda \log ^{2}(\kappa \lambda)\right)$ & $O\left(\kappa^{2} \lambda^{3}\right)$ & $O(n \log q)$ \\
$\mid$ par| & $O\left(\kappa^{3} \lambda \log ^{2}(\kappa \lambda)\right)$ & $O\left(\kappa^{3} \lambda^{5} \log (\kappa \lambda)\right)$ & $O\left(m_{r} \kappa n \log q\right)$ \\
\hline & & &
\end{tabular}


Shortest lattice Vector Problem with approximation factor $\gamma$ ) for the lattice $\mathcal{I}$, with $\gamma$ set at $\approx q^{3 / 8}$ to get a sufficiently short multiple of $g$. By the lattice reduction "rule of thumb," to make this cost $2^{\lambda}$, we need to set

$$
n=\Omega(\lambda \log q)
$$

When $\kappa=\operatorname{poly}(\log \lambda)$, the dimension $n$, encoding length $\mid$ enc $\mid$ and public parameters length |par| in our scheme GGHLite are all asymptotically close to optimal, namely quasi-linear in the security parameter $\lambda$, versus quadratic (resp. cubic and quintic) in $\lambda$ for GGH [9]. Thus we expect GGHLite's public parameters and encodings to be orders of magnitudes shorter than GGH for typical $\lambda \approx 100$.

Acknowledgments. We thank Vadim Lyubashevsky for useful discussions. This work has been supported in part by ERC Starting Grant ERC-2013-StG-335086LATTAC, an Australian Research Fellowship (ARF) from the Australian Research Council (ARC), and ARC Discovery Grants DP0987734 and DP110100628.

\section{References}

1. Agrawal, S., Gentry, C., Halevi, S., Sahai, A.: Discrete gaussian leftover hash lemma over infinite domains. In: Sako, K., Sarkar, P. (eds.) ASIACRYPT 2013, Part I. LNCS, vol. 8269, pp. 97-116. Springer, Heidelberg (2013)

2. Alperin-Sheriff, J., Peikert, C.: Circular and KDM security for identity-based encryption. In: Fischlin, M., Buchmann, J., Manulis, M. (eds.) PKC 2012. LNCS, vol. 7293, pp. 334-352. Springer, Heidelberg (2012)

3. Asharov, G., Jain, A., López-Alt, A., Tromer, E., Vaikuntanathan, V., Wichs, D.: Multiparty computation with low communication, computation and interaction via threshold FHE. In: Pointcheval, D., Johansson, T. (eds.) EUROCRYPT 2012. LNCS, vol. 7237, pp. 483-501. Springer, Heidelberg (2012)

4. Banerjee, A., Peikert, C., Rosen, A.: Pseudorandom functions and lattices. In: Pointcheval, D., Johansson, T. (eds.) EUROCRYPT 2012. LNCS, vol. 7237, pp. 719-737. Springer, Heidelberg (2012)

5. Boneh, D., Franklin, M.K.: Identity-based encryption from the Weil pairing. SIAM J. Comput. 32(3), 586-615 (2003)

6. Boneh, D., Silverberg, A.: Applications of multilinear forms to cryptography. Contemporary Mathematics 324, 71-90 (2003)

7. Coron, J.-S., Lepoint, T., Tibouchi, M.: Practical multilinear maps over the integers. In: Canetti, R., Garay, J.A. (eds.) CRYPTO 2013, Part I. LNCS, vol. 8042, pp. 476-493. Springer, Heidelberg (2013)

8. van Erven, T., Harremoës, P.: Rényi divergence and Kullback-Leibler divergence. CoRR, abs/1206.2459 (2012)

9. Garg, S., Gentry, C., Halevi, S.: Candidate multilinear maps from ideal lattices. In: Johansson, T., Nguyen, P.Q. (eds.) EUROCRYPT 2013. LNCS, vol. 7881, pp. 1-17. Springer, Heidelberg (2013)

10. Gentry, C.: Fully homomorphic encryption using ideal lattices. In: Proc. of STOC, pp. 169-178. ACM (2009)

11. Joux, A.: A one round protocol for tripartite Diffie-Hellman. In: Bosma, W. (ed.) ANTS-IV 2000. LNCS, vol. 1838, pp. 385-394. Springer, Heidelberg (2000) 
12. Lyubashevsky, V., Micciancio, D.: Generalized compact knapsacks are collision resistant. In: Bugliesi, M., Preneel, B., Sassone, V., Wegener, I. (eds.) ICALP 2006, Part II. LNCS, vol. 4052, pp. 144-155. Springer, Heidelberg (2006)

13. Lyubashevsky, V., Peikert, C., Regev, O.: On ideal lattices and learning with errors over rings. J. ACM 60(6), 43 (2013)

14. Micciancio, D., Goldwasser, S.: Complexity of lattice problems: a cryptographic perspective. Kluwer Academic Press (2002)

15. Micciancio, D., Regev, O.: Worst-case to average-case reductions based on Gaussian measures. SIAM J. Comput 37(1), 267-302 (2007)

16. Papamanthou, C., Tamassia, R., Triandopoulos, N.: Optimal authenticated data structures with multilinear forms. In: Joye, M., Miyaji, A., Otsuka, A. (eds.) Pairing 2010. LNCS, vol. 6487, pp. 246-264. Springer, Heidelberg (2010)

17. Regev, O.: Lecture notes of lattices in computer science, taught at the Computer Science Tel Aviv University, http://www.cims.nyu.edu/ regev/

18. Rényi, A.: On measures of entropy and information. In: Proc. of the Fourth Berkeley Symposium on Math. Statistics and Probability, vol. 1, pp. 547-561 (1961)

19. Rothblum, R.D.: On the circular security of bit-encryption. In: Sahai, A. (ed.) TCC 2013. LNCS, vol. 7785, pp. 579-598. Springer, Heidelberg (2013)

20. Rückert, M., Schröder, D.: Aggregate and verifiably encrypted signatures from multilinear maps without random oracles. In: Park, J.H., Chen, H.-H., Atiquzzaman, M., Lee, C., Kim, T.-h., Yeo, S.-S. (eds.) ISA 2009. LNCS, vol. 5576, pp. 750-759. Springer, Heidelberg (2009)

21. Sakai, R., Ohgishi, K., Kasahara, M.: Cryptosystems based on pairing. In: SCIS (2000)

22. Stehlé, D., Steinfeld, R.: Making NTRU as secure as worst-case problems over ideal. lattices. In: Paterson, K.G. (ed.) EUROCRYPT 2011. LNCS, vol. 6632, pp. 27-47. Springer, Heidelberg (2011)

23. Stehlé, D., Steinfeld, R.: Making NTRUEncrypt and NTRUSign as secure standard worst-case problems over ideal lattices, Full version of [22] (2013), http://perso.ens-lyon.fr/damien.stehle/NTRU.html 